\title{
Myopathological features in skeletal muscle of patients with chronic obstructive pulmonary disease
}

\author{
H.R. Gosker*, B. Kubat", G. Schaart", G.J. van der Vusse ${ }^{+}$, E.F.M. Wouters*, A.M.W.J. Schols*
}

Myopathological features in skeletal muscle of patients with chronic obstructive pulmonary disease. H.R. Gosker, B. Kubat, G. Schaart, G.J. van der Vusse, E.F.M. Wouters, A.M.W.J. Schols. (C) ERS Journals Ltd 2003.

ABSTRACT: Despite the fact that muscle weakness is a major problem in chronic obstructive pulmonary disease (COPD), detailed information on myopathological changes at the microscopic level in these patients is scarce, if indeed available at all.

Vastus lateralis biopsies of 15 COPD weight-stable patients (body mass index (BMI) $23.9 \pm 1.0 \mathrm{~kg} \cdot \mathrm{m}^{-2}$; fat-free mass index (FFMI) $17.2 \pm 1.7 \mathrm{~kg} \cdot \mathrm{m}^{-2}$ ) and 16 healthy agematched controls (BMI $26.3 \pm 0.8 \mathrm{~kg} \cdot \mathrm{m}^{-2}$; FFMI $19.6 \pm 2.2 \mathrm{~kg} \cdot \mathrm{m}^{-2}$ ) were evaluated. Histochemistry was used to evaluate myopathological features. Immunohistochemistry was used for the detection of macrophages and leukocytes, and active caspase 3 and terminal deoxynucleotidyl transferase deoxyuridine triphosphate (dUTP) nick-end labelling (TUNEL) as markers of apoptosis.

Fatty cell replacement and fibrosis were observed in both groups, the latter being slightly, but significantly, more pronounced in COPD. No differences between COPD and controls were found with respect to central nuclei, necrosis, regeneration, or fibre splitting. Signs of mitochondrial abnormalities were absent and normal numbers of inflammatory cells were found. Active caspase 3 positive myocytes were not observed and no difference was found in the number of TUNEL-positive myonuclei between controls and COPD patients (1.1\% versus $1.0 \%$, respectively). The cross-sectional area of type-IIX muscle fibres was smaller in COPD than in controls $(2,566$ versus $4,248 \mu^{2}$ ).

Except for the I to IIX shift in fibre types, the selective type-IIX atrophy and a slight accompanying increase in fibrosis and fat cell replacement in chronic obstructive pulmonary disease relative to age-matched controls, no other morphological abnormalities were observed in the muscle biopsies of chronic obstructive pulmonary disease patients. Also, in this group of clinically and weight stable chronic obstructive pulmonary disease patients, apoptosis appeared not to be involved in muscle pathology. Eur Respir J 2003; 22: 280-285.
Depts of *Pulmonology, ${ }^{*}$ Pathology and Movement Sciences, Nutrition Toxicology and Environment Research Institute Maastricht, and ${ }^{+}$Dept of Physiology, Cardiovascular Research Institute Maastricht, Maastricht University, Maastricht, the Netherlands.

Correspondence: H.R. Gosker

Dept of Respiratory Medicine

Maastricht University

P.O. Box 616

6200 MD Maastricht

The Netherlands

Fax: 31433875051

E-mail: H.Gosker@pul.unimaas.nl

Keywords: Chronic obstructive pulmonary disease

histological techniques

skeletal muscle

Received: February 52003

Accepted after revision: April 42003

This work was supported by a grant from the Netherlands Asthma Foundation (project number 96.16). Printing of the colour photograph was financially supported by Klinipath, the Netherlands.
Peripheral muscle weakness is a major problem in chronic obstructive pulmonary disease (COPD) $[1,2]$, contributing to exercise intolerance [3] and, hence, to a decreased health status [4]. The loss of muscle mass has been well described in these patients [1,2], but surprisingly, little data are reported regarding the morphology of limb muscles in COPD. First, muscle weakness has long been ignored, since studies focused primarily on the ventilatory limitations of exercise capacity. Secondly, those studies that did describe intrinsic muscular abnormalities in COPD tended to focus primarily on muscle fibre-type distributions and fibre sizes or dealt with molecular and biochemical alterations [5]. Thus, a complete pathological picture of the changes in limb muscle of patients with COPD is not yet available, whereas the microscopic examination of muscle biopsies is a first crucial step to enlarge the understanding of the underlying pathology in the peripheral muscles of COPD patients.

It is beyond the scope of this paper to describe all the different types of myopathies (for a review see PEARSON and YouNG [6]), but the parameters that are being used to screen the muscle may prove very helpful in the examination of myopathological features in the diseased muscle of COPD patients. In general, anybody suffering from severe peripheral muscle weakness will be referred to a pathologist or neurologist to assess the type and extent of the muscle pathology. The first step in the assessment of a peripheral muscle biopsy is to determine whether any abnormalities are present, and, if any, to determine if these changes are neurogenic, myopathic or inflammatory [7]. Neurogenic changes are characterised by fibre atrophy of both fibre types and the angular form of the atrophied fibres. Fibre type grouping occurs when reinnervation takes place. Nuclear clumps are the end products of very severe atrophy. In the mitochondrial enzyme staining moth-eaten fibres may be found. Fibrosis and replacement of myocytes by fat cells occurs in longstanding and substantial atrophy. In the second group, i.e. myopathies, increased variation of fibre size is not unusual, with atrophy and compensatory hypertrophy, the atrophied fibres are predominantly rounded and atrophy tends to be fibre-type specific (commonly type-I fibres), centralisation of the nuclei is often present and there is no fibre-type grouping. Fibrosis and fatty cell replacement of muscle tissue occurs especially in dystrophies (these could be defined as a severe and well defined subgroup of myopathies). The third group are the inflammatory muscle diseases, i.e. myositis, characterised by inflammatory infiltration and more 
or less pronounced necrosis, myolysis and regeneration of muscle fibres. Depending on the extent of muscle destruction, fibrosis may occur in the late stages.

The aim of the current study was to provide a detailed picture of skeletal muscle pathology in COPD, in order to gain more insight in the process of muscle wasting in these patients. For this purpose, muscle biopsies from the vastus lateralis of patients with COPD and healthy age-matched control subjects were examined. Microscopic evaluations were performed by means of standard histochemical techniques. Myopathological features were evaluated by an expert pathologist. In addition, inflammatory cells and apoptosis were evaluated immunohistochemically.

\section{Methods}

A group of 15 patients with moderate-to-severe airflow obstruction (aged 42-76 yrs) and 16 healthy age-matched volunteers (aged 60-70 yrs) was studied. All patients had COPD according to American Thoracic Society guidelines [8] and chronic airflow limitation, defined as measured forced expiratory volume in one second (FEV1) $<70 \%$ of reference FEV1. Furthermore, patients had irreversible obstructive airway disease $(<10 \%$ improvement of FEV1 predicted baseline after $\beta_{2}$-agonist inhalation). They were in a clinically stable condition and not suffering from a respiratory tract infection or an exacerbation of their disease at least 4 weeks prior to the study. Altogether, seven COPD patients were on a maintenance dose $\left(5-10 \mathrm{mg} \cdot \mathrm{day}^{-1}\right)$ of prednisolone. Exclusion criteria were malignancy, cardiac failure, distal arteriopathy, recent surgery, severe endocrine, hepatic or renal disorders, and use of anticoagulant medication. The healthy age-matched control subjects were volunteers recruited through an advertisement in a local newspaper. They underwent a physical examination by a physician to ensure that they were free from significant pulmonary or cardiac disease. Written informed consent was obtained from all subjects and the study was approved by the medical ethical committee of the University Hospital Maastricht (Maastricht, the Netherlands).

\section{Pulmonary function tests}

All patients and control subjects underwent spirometry to determine, amongst other measurements, FEV1, with the highest value from at least three technically acceptable assessments being used. Residual volume and intrathoracic gas volume were assessed by whole-body plethysmography and diffusion capacity for carbon monoxide was measured by using the single-breath method (Masterlab; Jaeger, Wurzburg, Germany). All values obtained were expressed as percentage of the predicted value [9]. Arterial oxygen tension was determined (ABL 330; Radiometer, Copenhagen, Denmark) in a blood sample from the radial artery while subjects breathed room air.

\section{Assessment of body composition}

Body height was determined to the nearest $0.5 \mathrm{~cm}$ and body weight was assessed to the nearest $0.1 \mathrm{~kg}$ while subjects wore light clothing and no shoes. Fat-free mass (FFM) was determined by scanning subjects on a DPX bone densitometer (Lunar Radiation Corporation, Madison, WI, USA) as described previously [10]. Weight parameters were adjusted for body surface, resulting in the BMI and FFM index (FFMI) [11].

\section{Assessment of muscle function}

Isometric handgrip strength was assessed in all subjects by using a Harpenden handgrip dynamometer (Yamar; Preston, Jackson, MI, USA). Linear isokinetic muscle function of the lower limbs was assessed by a multijoint dynamometer device (Aristokin; Lode, Groningen, the Netherlands) at $20 \mathrm{~cm} \cdot \mathrm{s}^{-1}$ and at $60 \mathrm{~cm} \cdot \mathrm{s}^{-1}$ as described previously [2]. At a speed of $20 \mathrm{~cm} \cdot \mathrm{s}^{-1}$, the maximal power generated by the legs mainly determined the outcome of performance. At a speed of $60 \mathrm{~cm} \cdot \mathrm{s}^{-1}$ performance is also influenced by muscle coordination. Before testing, each subject was familiarised in a standardised way with the equipment and the requested movement.

\section{Collection and processing muscle tissue}

Postabsorptive muscle biopsies of the vastus lateralis were obtained by the needle biopsy technique [12]. The specimen was frozen in melting isopentane precooled in liquid nitrogen and stored at $-35^{\circ} \mathrm{C}$. The examination of muscle morphology was performed on serial cryostat cross-sections, of $10 \mu \mathrm{m}$ thickness. General muscle architecture and fibre morphology were shown by routine haematoxylin-eosin (H\&E) staining or van Gieson staining (for additional collagen staining). Muscle-fibre typing and distribution (fibre types I, IIA and IIX) were evaluated by myofibrillar adenosine triphosphotease (mATPase) staining and immunohistochemistry as described previously. The combined staining results of the three different $\mathrm{mATPase}$ stainings (one with pre-incubation at $\mathrm{pH} 4.4$, one at $\mathrm{pH} \mathrm{10.4}$, and double pre-incubation at $\mathrm{pH} 10.4$ followed by 4.6) with three antibodies against myosine heavy chain (MyHC) isoforms (anti-type I MyHC (mAb 219-1D1), anti-type IIA MyHC (mAb 333-7H1) and anti-types IIA+IIX MyHC (mAb 332-3D4)) and at least 100 (but mostly up to 200) fibres were analysed [13]. Fibre crosssectional area (CSA) and roundness (values ranging $0-1$, with 1 being perfectly round) were measured with an interactive image analysis system (Leica QWin Image Analysis System; Leica Microsystems BV, Rijswijk, the Netherlands) [14]. Cytochrome c oxidase (COX) and succinate dehydrogenase (SDH) staining was performed as described previously [13]. The altered muscle fibre-type distribution, metabolic profile and reduced fibre type IIX CSA shown in table 1 have been reported earlier [13, 14]. Deoxyribonucleic acid (DNA) fragmentation in muscle-fibre nuclei was detected by terminal deoxynucleotidyl transferase dUTP nick end labelling (TUNEL) after pretreatment with proteinase $\mathrm{K}$ for $5 \mathrm{~min}$, followed by diaminobenzidine staining with a peroxidase conjugated antibody (TdT-FragEL ${ }^{\mathrm{TM}}$ DNA Fragmentation Detection Kit, Oncogene Research Products, Boston, MA, USA). Altogether, 300-500 myocyte nuclei were counted using a grid and the percentage of positive nuclei was determined. A common problem of the TUNEL reaction is the weak background staining of nuclei. Therefore as TUNEL-positive control, muscle sections were treated with $1 \mathrm{mg} \cdot \mathrm{mL}^{-1}$ deoxyribonucleic (DNase) I, to obtain DNA fragmentation. The staining intensity in these nuclei served as positive controls. Active caspase 3 was evaluated by immunostaining with the polyclonal anti-active caspase 3 antibody CM1 (Idun Pharmaceuticals, La Jolla, CA, USA). Mouse monoclonal antibodies were used for identification of leukocytes (anti-leukocyte common antigen CD45, M0701; Dako Ltd, High Wycombe, UK) and macrophages (anti-CD68, M0814; Dako). Primary antibody binding was detected with the StreptABComplex/HRP method (K0377; Dako) and staining with diaminobenzidine. Cryosections of a human malignant sarcoma and human tonsil were 
Table 1.-Subject characteristics, lung function and muscle biopsy data

Controls COPD

\begin{tabular}{|c|c|c|}
\hline \multicolumn{3}{|l|}{ General characteristics } \\
\hline $\mathrm{M}: \mathrm{F}$ & $14: 2$ & $12: 3$ \\
\hline Age yrs & $64 \pm 3$ & $67 \pm 9$ \\
\hline $\mathrm{BMI} \mathrm{kg} \cdot \mathrm{m}^{-2}$ & $26.3 \pm 3.3$ & $23.9 \pm 4.0$ \\
\hline FFM kg & $58.2 \pm 9.0$ & $48.8 \pm 6.9 * *$ \\
\hline FFMI $\mathrm{kg} \cdot \mathrm{m}^{-2}$ & $19.6 \pm 2.2$ & $17.2 \pm 1.7 * *$ \\
\hline FEV1\% pred & $109 \pm 17.2$ & $42 \pm 13.9 * * *$ \\
\hline $\mathrm{Pa}, \mathrm{O}_{2} \mathrm{kPa}$ & $11.8 \pm 1.6$ & $9.9 \pm 1.2 * * *$ \\
\hline$D \mathrm{~L}, \mathrm{CO} \%$ pred & $123 \pm 23$ & $63 \pm 24 * * *$ \\
\hline RV \% pred & $114 \pm 15$ & $167 \pm 32 * * *$ \\
\hline ITGV $\%$ pred & $108 \pm 17$ & $144 \pm 22 * * *$ \\
\hline \multicolumn{3}{|l|}{ Muscle function } \\
\hline Leg power at $20 \mathrm{~cm} \cdot \mathrm{s}^{-1} \mathrm{~W}$ & $265 \pm 62$ & $203 \pm 88 *$ \\
\hline Leg power at $60 \mathrm{~cm} \cdot \mathrm{s}^{-1} \mathrm{~W}$ & $561 \pm 154$ & $363 \pm 145^{* *}$ \\
\hline Handgrip strength kg & $43 \pm 8$ & $31 \pm 8 * * *$ \\
\hline \multicolumn{3}{|l|}{ Muscle biopsy } \\
\hline Proportion of type-I fibres $\%$ & $43.4 \pm 12.6$ & $19.4 \pm 14.0^{* * *}$ \\
\hline Proportion of type-IIA fibres $\%$ & $29.4 \pm 12.1$ & $34.8 \pm 11.9$ \\
\hline Proportion of type-IIX fibres $\%$ & $27.2 \pm 12.3$ & $45.8 \pm 18.9^{* * *}$ \\
\hline CSA of type-IIX fibres $\mu \mathrm{m}^{2}$ & $4248 \pm 1300$ & $2566 \pm 1137 * *$ \\
\hline
\end{tabular}

Data are presented as mean \pm SD. M: male; F: female; BMI: body mass index; FFM: fat-free mass; FFMI: fat-free mass index; FEV1: forced expiratory volume in one second; \% pred; \% predicted; $\mathrm{Pa}, \mathrm{O}_{2}$ : arterial oxygen tension; $D \mathrm{~L}, \mathrm{CO}$ : diffusion capacity for carbon monoxide; $\mathrm{RV}$ : residual volume; ITGV: intrathoracic gas volume; CSA: cross-sectional area. Significance of difference compared with controls. ${ }^{*}$ : $\mathrm{p}<0.05$; **: $\mathrm{p}<0.01 ; * * *: \mathrm{p}<0.001$. Control: $\mathrm{n}=16$; COPD: $\mathrm{n}=15$.

used as a positive control for active caspase 3, and leukocytes/macrophages, respectively. In TUNEL, active caspase 3 , leukocyte, and macrophage staining nuclei were counterstained with haematoxylin. The following features were evaluated semi-quantitatively: 1) inflammatory infiltrates, fibrosis, fatty cell replacement, fibre splitting, vacuoles, necrosis, regeneration, hyaline and ring fibres, and central nuclei (H\&E); 2) mitochondrial rearrangements, such as subsarcolemmal accumulation, targets, moth-eaten fibres and cores (COX and SDH); and 3) fibre-type distribution and grouping, and atrophied/hypertrophied fibres (mATPase). This screening was performed in a blinded manner by an expert pathologist who assigned scores ranging $0-5$ indicating the severity of each feature, 0 for the absence of the feature, 5 for severe abnormalities.

\section{Statistical analysis}

Data were analysed with the unpaired t-test (corrected for unequal variances if required), Pearson Chi-squared test, or the Pearson correlation test [15]. Analysis of covariance with sex as covariate was used to compare leg power between COPD and controls. Data are presented as mean \pm SD. A twotailed probability value of $<0.05$ was considered statistically significant.

\section{Results}

Subject characteristics are shown in table 1. There were no differences in sex or age between the groups. FFMI was significantly lower in COPD patients than in controls, despite a similar BMI. Isokinetic leg power was significantly lower in COPD patients than in controls, at both speeds. Handgrip strength was also lower in COPD patients compared with healthy subjects. The proportions of type-I fibres (fibres with high oxidative capacity) were markedly lower in COPD patients, whereas the fibre type-IIX proportion was higher compared with healthy subjects. Fibre-type IIX CSA was significantly smaller in COPD patients.

Frequencies of the severity scores in controls versus COPD patients are shown in table 2 . No scores $>2$ for severity (on a scale of $0-5$ ) were obtained for any of the semiquantitatively

Table 2. - Severity score frequencies of myopathological features

\begin{tabular}{|c|c|c|c|c|c|c|c|}
\hline \multirow[t]{3}{*}{ Pathological feature } & \multicolumn{6}{|c|}{ Severity score } & \multirow[t]{3}{*}{$\mathrm{p}$-value } \\
\hline & \multicolumn{2}{|c|}{0} & \multicolumn{2}{|c|}{1} & \multicolumn{2}{|c|}{2} & \\
\hline & Control & COPD & Control & COPD & Control & COPD & \\
\hline Atrophied fibres & 8 & 1 & 8 & 5 & 0 & 9 & $<0.001$ \\
\hline Hypertrophied fibres & 15 & 10 & 1 & 5 & 0 & 0 & \\
\hline Fibre type grouping & 4 & 5 & 11 & 9 & 1 & 1 & \\
\hline Fibre splitting & 13 & 13 & 3 & 2 & 0 & 0 & \\
\hline Fibrosis perimysial & 12 & 10 & 4 & 2 & 0 & 3 & \\
\hline Fibrosis endomysial & 8 & 1 & 8 & 14 & 0 & 0 & $<0.01$ \\
\hline Fat cells & 12 & 8 & 3 & 6 & 1 & 1 & \\
\hline Inflammatory cells & 16 & 12 & 0 & 3 & 0 & 0 & \\
\hline Central nuclei & 15 & 13 & 1 & 2 & 0 & 0 & \\
\hline Necrotising fibres & 16 & 12 & 0 & 3 & 0 & 0 & \\
\hline Regenerating fibres & 13 & 8 & 3 & 7 & 0 & 0 & \\
\hline Cores & 16 & 15 & 0 & 0 & 0 & 0 & \\
\hline Moth-eaten fibres & 16 & 12 & 0 & 3 & 0 & 0 & \\
\hline Targets & 16 & 15 & 0 & 0 & 0 & 0 & \\
\hline Hyaline fibres & 16 & 15 & 0 & 0 & 0 & 0 & \\
\hline Ring fibres & 16 & 14 & 0 & 1 & 0 & 0 & \\
\hline
\end{tabular}

Data are presented as n. Frequencies for severity scores 3-5 are not shown, since none of the subjects had scores $>2$ for any of the features. Atrophy and hypertrophy: abnormal fibre size and/or shape; fibre-type grouping: replacement of the normal mosaic pattern by groups of histochemically uniform fibre types; inflammatory cells: macrophages and leukocytes; cores: zones devoid of oxidative enzyme activity staining; moth-eaten: patchy staining due to the disruption of the intermyofibrillar; targets: unreactive core surrounded by a darker staining rim; hyline fibres: rounded, heavily haematoxylin and eosin stained fibres (due to hypercontraction of myofibrils); ring fibres: dense subsarcolemmal staining. Significance of difference compared to controls (based on Pearson Chi-squared). Control: $n=16$; COPD: $n=15$. 
screened myopathological features either in COPD patients or in healthy subjects. Normal amounts of macrophages and leukocytes were found, randomly scattered between fibres in control subjects as well as in COPD patients. There were no clear signs of excessive necrosis and/or regeneration in either group. There were no signs of mitochondrial or cytoskeletal rearrangements, such as moth-eaten fibres, cores, target fibres, hyaline fibres or ring fibres (apart from the higher proportion of fibres deficient for COX and SDH in COPD) in either group. In both patients and controls a mild replacement of muscle by fat cells and fibrotic material was observed (fig. 1a and b), the latter being slightly more pronounced in COPD patients (Chi-squared=7.06; $\mathrm{p}=0.008$ ). Atrophy of muscle fibres was clearly more pronounced in COPD patients (Chi-squared=15.12; $\mathrm{p}=0.001$ ). In both groups, the atrophied fibres were randomly scattered and mainly had angulated shapes (fig. 1c), although rounded fibres were also observed. The roundness of type-IIX fibres was also assessed, and was less in COPD compared with controls $(0.70 \pm 0.07$ versus $0.78 \pm 0.06 ; \mathrm{p}=0.007)$.
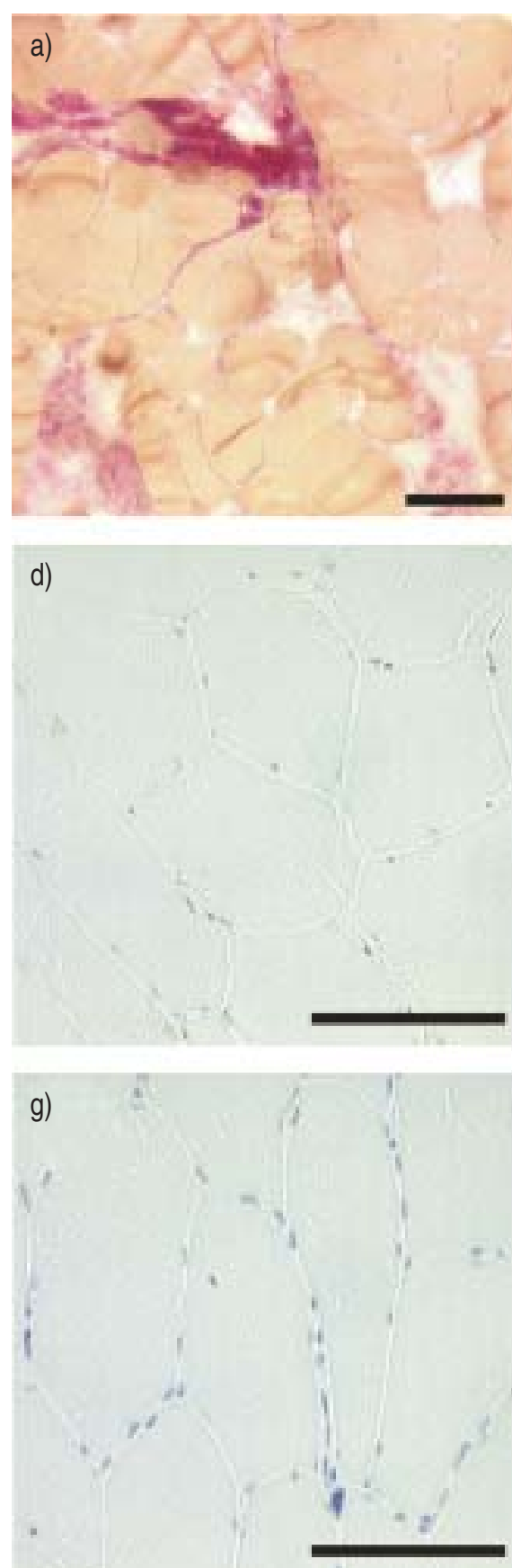


Fig. 1.-a) Van Gieson stain showing increased amounts of connective tissue (collagen stains red). Both perimysial and endomysial fibrosis is present. b) Haematoxylin-eosin stain showing fat cell replacement. The fat cells appear as "holes", because of lack of staining. c) Myofibrillar adenosine triphosphatease stain (double pre-incubation at $\mathrm{pH} 10.4$ followed by 4.6) showing small and angulated dark fibres that are atrophied type-IIX fibres. Note the large variation in fibre sizes. d) Representative transferase deoxyuridine triphosphate nick-end labelling (TUNEL) stain of a healthy control. e) Representative TUNEL stain of a chronic obstructive pulmonary disease (COPD) patient. f) Positive control for TUNEL staining (a deoxyribonucleic acid I streated section). g) Representative caspase 3 stain of a healthy control. h) Representative caspase 3 stain of a COPD patient. i) Positive control for caspase 3 staining (a human malignant sarcoma). Internal scale bars $=100 \mu \mathrm{m}$. 
DNA fragmentation was assessed with TUNEL staining (fig. 1d-f). The proportion of positive nuclei was not different between controls and COPD patients $(1.1 \pm 1.6 \%$ versus $1.0 \pm 0.8 \%$ ). The proportion of TUNEL-positive nuclei did not correlate with fibre CSA or with FFM in COPD patients or controls. The presence of active caspase 3 as a marker of apoptosis was also examined, but positive fibres were absent in both groups (fig. 1g-i).

Altogether, seven of the 15 COPD patients were on a maintenance dose of prednisolone during the study. However, there were no differences for any of the myopathological features examined in the current study between the patients who received corticosteroids and nonreceivers. Also, the proportion of TUNEL-positive nuclei was not different between receivers and nonreceivers $(1.1 \pm 0.8 \%$ versus $0.9 \pm 0.9 \%$, respectively).

\section{Discussion}

In this study the "classical" myopathological features in the vastus lateralis of healthy controls and patients with COPD was assessed. The COPD patients had reduced muscle mass and suffered from muscle weakness. Compared with healthy age-matched controls, a I to IIX shift in fibre types and marked fibre-type IIX atrophy was present as reported previously $[13,14]$. There were no other clear morphological differences between controls and patients, except for a slight increase of intramuscular fibrosis and fat-cell replacement in COPD. This can probably be explained by the higher degree of fibre atrophy in the patients, since the presence of increased connective tissue and fat cells is common for the severely atrophying muscle [6]. Indeed, some degree of fibrosis and fatty cell replacement is not uncommon for the skeletal muscle of the elderly, which is probably associated with the age-related atrophy of type-II fibres [16]. The observation of rounded fibres secondary to angulated fibres, suggests that the observed muscle atrophy is slowly progressing and longstanding. Therefore, the abnormalities found can probably be attributed to the general pathology in the elderly, for example caused by physical inactivity or microangiopathy. Microangiopathy in peripheral neuropathy (examined in nerve biopsies) has been reported for COPD and the involvement of hypoxia was suggested [17]. OzGE et al. [18] also found a correlation between the degree of neuropathy (functionally assessed) and hypoxaemia in COPD. However, patients in the current study were not extremely hypoxic and it is therefore worth examining these myopathological features in patients with severe hypoxaemia.

The specific form of fibre-type IIX atrophy excludes a predominant involvement of physical inactivity, because a more generalised, nonfibre type specific atrophy would be expected [6, 7]. DECRAMER et al. [19] showed selective fibre type-II atrophy in COPD patients with diagnosed steroidinduced myopathy. In that study affected fibres were also angulated and increased connective tissue was observed. In contrast to their data, no increase in numbers of necrotic fibres and central nuclei was found in this study. Furthermore, no differences were found between COPD patients who received (low doses of) prednisolone and those who did not. The authors also had no previous evidence for the involvement of corticosteroids in fibre type-IIX atrophy [14].

The aetiology of fibre type-IIX atrophy is still not understood completely, but its involvement in the loss of muscle mass and, hence, its effect on muscle strength in COPD is obvious [14]. Although the COPD patients in the current study were not cachectic, they were characterised by a reduced FFMI relative to the control group. Atrophy may not be the only factor that is associated with the loss of muscle mass. Necrosis and apoptosis (i.e. "programmed" cell death) are two other processes that may lead to the reduction of muscle mass through the disappearance of muscle cells. The present data suggest that necrosis is not involved, which is underscored by the fact that the authors did not observe any widespread infiltrates of immune cells that normally accompany necrosis [20]. The authors found that only $\sim 1 \%$ of all myocyte nuclei was TUNEL-positive, for controls as well as for COPD patients. However, using TUNEL for the detection of apoptosis should be interpreted with caution, since nonspecific DNA fragmentation may also be detected [21]. Therefore, the authors also immunostained for active caspase 3 , an established marker for apoptosis [21], and in neither controls nor patients cytoplasmatic immunostaining for active caspase 3 was found. The data in the current study thus suggest that apoptosis of muscle fibres does not occur, certainly not to a higher degree in COPD patients compared with controls. However, despite the authors reservations regarding the TUNEL data, few positive nuclei in COPD patients as well as in healthy subjects were found. Since myocytes are multi-nucleated, apoptotic nuclei may well be important to adjust the number of myonuclei in fibres during atrophying conditions [22]. A relationship between fibre CSA and the number of TUNEL-positive myonuclei has indeed been shown in chronic heart failure [23]. However, the authors did not find this relationship in COPD, which may be related to the low frequency of TUNEL-positivity or because the patients in the current study were in a weight-stable condition and may be less underweight. Alternatively, since apoptosis is a very dynamic process and the progress of muscle wasting is relatively slow, very small differences may be expected between patients and controls. Therefore, it is possible that the current methodology is not sufficient to detect those potential differences.

Muscle wasting has convincingly been associated with systemic inflammation in COPD [24-26]. Also, it is feasible that circulating inflammatory cells infiltrate into the muscle in a similar way to bronchial inflammatory cell infiltration. Therefore, the presence of inflammatory cells in the wasting muscle can be expected. However, the authors did not find abnormal numbers of macrophages and leukocytes in the muscle tissue of COPD patients. This indicates that intramuscular inflammatory cells are most likely not the source of inflammatory mediators that affect the muscle. It should be noted that there is a small probability that magnitude of inflammatory cell infiltration into the muscle could have been reduced by corticosteroid administration. However, as discussed above, only one-half of the patients received this medication and the doses were low. Alternatively, circulating cytokines and chemokines or mediators produced by local endothelial cells or even the myocytes themselves, may be involved in the association between inflammation and skeletal muscle wasting. However, this assumption requires further investigation, which was beyond the scope of the present study.

In summary, this is the first time that peripheral muscle weakness in chronic obstructive pulmonary disease has been approached from a myopathological perspective using a broad set of standard histochemical techniques. Apart from the marked fibre type-IIX atrophy in chronic obstructive pulmonary disease, the results show little difference between patients with chronic obstructive pulmonary disease and healthy age-matched control subjects. In addition to the I to IIX shift in fibre types and selective type-IIX atrophy in chronic obstructive pulmonary disease, a slight increase in fibrosis and fat-cell replacement, which probably accompanies the fibre atrophy, was observed in these patients. No other clear features of muscle pathologies were observed, nor was there an increase in the amount of intramuscular 
inflammatory cells. Furthermore, there were no indications that necrosis and apoptosis are involved in muscle pathology. Future studies are required to determine potential differences in muscle pathology in the subgroups of severe hypoxic patients and cachectic chronic obstructive pulmonary disease patients (characterised by weight loss and disproportionate wasting of fat-free mass) and to further describe the mechanisms underlying skeletal muscle fibre atrophy.

Acknowledgements. The authors would like to thank M. Hesselink for general assistance and E. Dumont for performing the caspase 3 staining.

\section{References}

1. Bernard S, LeBlanc P, Whittom F, et al. Peripheral muscle weakness in patients with chronic obstructive pulmonary disease. Am J Respir Crit Care Med 1998; 158: 629-634.

2. Engelen MP, Schols AM, Does JD, Wouters EF. Skeletal muscle weakness is associated with wasting of extremity fatfree mass but not with airflow obstruction in patients with chronic obstructive pulmonary disease. Am J Clin Nutr 2000; 71: 733-738.

3. Schols AM, Mostert R, Soeters PB, Wouters EF. Body composition and exercise performance in patients with chronic obstructive pulmonary disease. Thorax 1991; 46: 695-699.

4. Wood PH. Appreciating the consequences of disease: the international classification of impairments, disabilities, and handicaps. WHO Chron 1980; 34: 376-380.

5. Gosker HR, Wouters EF, van der Vusse GJ, Schols AM. Skeletal muscle dysfunction in chronic obstructive pulmonary disease and chronic heart failure: underlying mechanisms and therapy perspectives. Am J Clin Nutr 2000; 71: 1033-1047.

6. Pearson AM, Young RB. Diseases and disorders of muscle Adv Food Nutr Res 1993; 37: 339-423.

7. Dubowitz V. Muscle Biopsy. A Practical Approach. London, Baillière Tindall, 1985.

8. American Thoracic Society. Standards for the diagnosis and care of patients with chronic obstructive pulmonary disease (COPD) and asthma. Am Rev Respir Dis 1987; 136: 225-244.

9. Quanjer P, Tammeling GJ, Cotes JE, Pedersen OF, Peslin R, Yernault JC. Standardized lung function testing. Eur Respir $J$ 1993; 6: 5-40

10. Engelen MPKJ, Schols AMWJ, Heidendal GAK, Wouters EFM. Dual-energy X-ray absorptiometry in the clinical evaluation of body composition and bone mineral density in patients with chronic obstructive pulmonary disease. $\mathrm{Am}$ J Clin Nutr 1998; 68: 1298-1303.

11. VanItallie TB, Yang MU, Heymsfield SB, Funk RC, Boileau RA. Height-normalized indices of the body's fat-free mass and fat mass: potentially useful indicators of nutritional status. Am J Clin Nutr 1990; 52: 953-959.

12. Bergstrom L. Muscle electrolytes in man. Determination by neutron activation analysis on needle biopsy specimens A study on normal subjects, kidney patients, and patients with chronic diarrhoea. Scand J Clin Lab Invest 1962; 68: $1-110$.

13. Gosker HR, van Mameren H, van Dijk PJ, et al. Skeletal muscle fibre type shifting and metabolic profile in patients with COPD. Eur Respir J 2002; 19: 617-626.

14. Gosker HR, Engelen MPKJ, van Mameren H, et al. Muscle fiber type IIX atrophy is involved in the loss of fat-free mass in chronic obstructive pulmonary disease. Am J Clin Nutr 2002; 76: 113-119.

15. Altman DG, Gore SM, Gardner MJ, Pocock SJ. Statistical guidelines for contributors to medical journals. $\mathrm{Br}$ Med $J$ (Clin Res Ed) 1983; 286: 1489-1493.

16. O'Rourke KS. Myopathies in the elderly. Rheum Dis Clin North Am 2000; 26: 647-672.

17. Stoebner P, Mezin P, Vila A, Grosse R, Kopp N, Paramelle B. Microangiopathy of endoneurial vessels in hypoxemic chronic obstructive pulmonary disease (COPD). A quantitative ultrastructural study. Acta Neuropathol (Berl) 1989; 78: $388-395$.

18. Ozge A, Atis S, Sevim S. Subclinical peripheral neuropathy associated pulmonary disease. Electromyogr Clin Neurophysiol 2001; 41: 185-191.

19. Decramer M, de Bock V, Dom R. Functional and histologic picture of steroid-induced myopathy in chronic obstructive pulmonary disease. Am J Respir Crit Care Med 1996; 153: $1958-1964$

20. McConkey DJ. Biochemical determinants of apoptosis and necrosis. Toxicol Lett 1998; 99: 157-168.

21. de Boer RA, van Veldhuisen DJ, van der Wijk J, et al. Additional use of immunostaining for active caspase 3 and cleaved actin and PARP fragments to detect apoptosis in patients with chronic heart failure. J Card Fail 2000; 6 : 330-337.

22. Allen DL, Roy RR, Edgerton VR. Myonuclear domains in muscle adaptation and disease. Muscle Nerve 1999; 22: 13501360.

23. Vescovo G, Volterrani M, Zennaro R, et al. Apoptosis in the skeletal muscle of patients with heart failure: investigation of clinical and biochemical changes. Heart 2000; 84 431-437.

24. Eid AA, Ionescu AA, Nixon LS, et al. Inflammatory response and body composition in chronic obstructive pulmonary disease. Am J Respir Crit Care Med 2001; 164 1414-1418.

25. Di Francia M, Barbier D, Mege JL, Orehek J. Tumor necrosis factor-alpha levels and weight loss in chronic obstructive pulmonary disease. Am J Respir Crit Care Med 1994; 150: 1453-1455

26. Schols AM, Buurman WA, Staal van den Brekel AJ, Dentener MA, Wouters EF. Evidence for a relation between metabolic derangements and increased levels of inflammatory mediators in a subgroup of patients pulmonary disease. Thorax 1996; 51: 819-824. 